\title{
ANDES

www.scielo.cl

\section{Caracterización microbiológica y de susceptibilidad antimicrobiana de las infecciones asociadas a neutropenia febril en pacientes hemato-oncológicos pediátricos}

\author{
Microbiological characterization and antimicrobial susceptibility pattern of infections \\ associated with febrile neutropenia in pediatric hemato-oncological patients
}

Bello-Suárez AK ${ }^{\mathrm{a}}$, Cuesta-Armesto $\mathrm{MH}^{\mathrm{a}}$, Díaz-Rojas MJ ${ }^{\mathrm{a}}$, Leidy Mendoza-Uribe ${ }^{\mathrm{a}}$, Sarmiento-Wilches PE ${ }^{\mathrm{a} b}$

aUniversidad Industrial de Santander. Bucaramanga, Colombia

${ }^{\text {b} C l i ́ n i c a ~ M a t e r n o ~ I n f a n t i l ~ S a n ~ L u i s . ~ B u c a r a m a n g a, ~ C o l o m b i a ~}$

Recibido: 15 de enero de 2021; Aceptado: 16 de agosto de 2021

¿Qué se sabe del tema que trata este estudio?

La documentación microbiológica es una herramienta fundamental para una terapia antibiótica empírica oportuna, apropiada y racional en la neutropenia febril. La vigilancia epidemiológica es heterogénea y en ocasiones limitada en Latinoamérica, lo que condiciona a mayores complicaciones infecciosas en esta población.
¿Qué aporta este estudio a lo ya conocido?

El presente trabajo, al ser representativo de la población regional, brinda una base del comportamiento microbiológico en la neutropenia febril, tanto a nivel local, nacional e incluso en comunidades latinoamericanas con condiciones poblacionales similares, para un uso adecuado de los antibióticos.

\section{Resumen}

La neutropenia febril (NF) es la urgencia hemato-oncológica de mayor frecuencia, morbilidad y mortalidad en pediatría. Objetivo: Describir la caracterización microbiológica y de la susceptibilidad antimicrobiana de las infecciones asociadas a NF en pacientes hemato-oncológicos pediátricos. Pacientes y Método: Estudio cohorte retrospectivo en pacientes entre 1 mes y 18 años de edad con patología onco-hematológica según códigos CIE-10, hospitalizados en un centro de tercer nivel de atención de Colombia. Basados en las historias clínicas del período 2013-2017 se detectaron los episodios de NF y se describieron los microorganismos aislados y su patrón de susceptibilidad. Las pruebas de identificación bioquímica y de susceptibilidad a los antimicrobianos fueron realizadas con el sistema automatizado Dade Behring Microscan ${ }^{\circledR}$. La clasificación de microorganismo resistente se realizó teniendo en cuenta la Concentración Mínima Inhibitoria (CMI) y la interpretación realizada por el laboratorio de acuerdo a los puntos de corte de las recomendaciones del Instituto de Estándares Clínicos y de Laboratorio. Resultados: De 130 pacientes el 14,7\% de los cultivos obtenidos fueron po-
Palabras clave: Neutropenia Febril; Infección Bacteriana; Cáncer; Microorganismos; Bacteremia 
sitivos. Se documentó infección del torrente sanguíneo en el 17,5\% de los episodios. Los microorganismos aislados fueron principalmente Bacilos Gram-negativos (BGN) (75,8\%); las Enterobacterias (EB) fueron las más frecuentes, encabezadas por Klebsiella pneumoniae, Escherichia coli, seguidas de Pseudomonas aeruginosa y Staphylococcus coagulasa negativos (SCN). De las EB, el 40,5\% mostraron resistencia a Piperacilina Tazobactam (TZP), 33,3\% a Cefepime (FEP) y 8,2\% a Meropenem (MEM). De acuerdo al patrón de resistencia antimicrobiana, se documentó que, de los cultivos positivos, el 16,4\% de EB tenían patrón betalactamasas de espectro extendido (BLEE) y 5\%, patrón sugestivo de carbapenemasas (PC). Todos los Cocos Gram-positivos (CGP) fueron sensibles a Vancomicina (VAN). Conclusión: En los pacientes estudiados, los microorganismos patógenos predominantes fueron los Gram-negativos con índices de resistencia similares a países en vías de desarrollo.

\begin{abstract}
Febrile neutropenia (FN) is the most frequent hemato-oncological emergency, with high morbidity and mortality in pediatrics. The objective of the study was the microbiological characterization and antimicrobial susceptibility of infections associated with FN in pediatric hemato-oncological patients. Patients and Method: Retrospective cohort study with patients aged between 1 month and 18 years, with onco-hematological pathology according to ICD-10 codes, hospitalized in a tertiary healthcare center in Bucaramanga, Colombia. Based on the medical records of the period 2013-2017, the episodes of FN were identified, and the isolated microorganisms and their susceptibility pattern were described. Biochemical identification and antimicrobial susceptibility testing were performed with the Dade Behring Microscan ${ }^{\circledR}$ automated system. The resistant microorganism classification was performed based on the Minimum Inhibitory Concentration (MIC) and the interpretation of the laboratory according to the cut-off points of the Clinical and Laboratory Standards Institute recommendations. Results: Of 130 patients, $14.7 \%$ of the cultures obtained were positive. Bloodstream infection was observed in $17.5 \%$ of the episodes. The isolated microorganisms were mainly Gram-negative bacteria (75.8\%). Enterobacteriaceae (EB) were the most frequent, led by Klebsiella pneumoniae, Escherichia coli, followed by Pseudomonas aeruginosa and coagulase-negative Staphylococci. Of the EBs, $40.5 \%$ showed resistance to Piperacillin/Tazobactam, 33.3\% to Cefepime, and 8.2\% to Meropenem. According to the antimicrobial resistance pattern, it was observed that $16.4 \%$ of the positive EB cultures had an extended-spectrum beta-lactamase pattern and $5 \%$ a pattern suggestive of carbapenemases. All Gram-positive cocci were sensitive to Vancomycin. Conclusion: In the studied patients, the predominant pathogenic microorganisms were Gram-negative ones with resistance indices similar to those of developing countries.
\end{abstract}

\section{Keywords:}

Febrile Neutropenia;

Bacterial Infection;

Cancer;

Microorganisms;

Bloodstream Infection

\section{Introducción}

El cáncer es la primera causa de muerte no accidental en niños de países en desarrollo, siendo la NF una de las urgencias más frecuentes y de mayor morbilidad y mortalidad en pacientes con patología hemato-oncológica. Con el fin de controlar las infecciones bacterianas invasivas, la instauración de antibioticoterapia de amplio espectro, previa toma de cultivos, debe ser de inicio rápido. Según las guías de manejo, la documentación microbiológica es una herramienta invaluable para realizar una terapia empírica racional acorde a la realidad institucional; ya que la dinámica evolutiva de los microorganismos, el uso inapropiado e irracional de antibióticos de amplio espectro con propósitos profilácticos o terapéuticos, las superinfecciones, la migración de nuevos microorganismos y cepas entre instituciones, son factores que predisponen a cambios en la etiología y el patrón de susceptibilidad antimicrobiana $^{1-4}$.

El objetivo del estudio fue describir la caracterización epidemiológica, microbiológica y del patrón de susceptibilidad antimicrobiana de las infecciones asociadas a NF en pacientes hemato-oncológicos pediátricos en Bucaramanga, Colombia.

\section{Pacientes y Método}

Estudio descriptivo de tipo cohorte retrospectivo, que incluyó pacientes entre 1 mes y 18 años con patología onco-hematológica, según código CIE-10, que estaban hospitalizados en la Clínica Materno Infantil San Luis (Bucaramanga, Colombia). Se revisaron las historias clínicas del período 2013-2017, para detectar los episodios de NF, recolectar los datos en el progra- 
ma REDCap y realizar la descripción de los microorganismos aislados y su patrón de susceptibilidad.

Fueron incluidos todos los pacientes menores de 18 años, con diagnóstico oncológico o hematológico susceptibles de presentar NF (todo aquel con diagnóstico de patología hematológica y oncológica definidos de alto riesgo en la historia clínica, con progresión rápida de la enfermedad, o linfomas de estadios avanzados), hospitalizados en el periodo estudiado. Se excluyeron todos los recién nacidos y aquellos pacientes cuya neutropenia febril fuera secundaria a patologías no hematológicas ni oncológicas (reumatológicas, nutricionales, metabólicas, endocrinológicas, infecciosas, farmacológicas diferentes a antineoplásicos e inmunológicas).

Se definió como episodio de NF a la documentación en la historia clínica de una temperatura inicial $\geq 38^{\circ} \mathrm{C}$ junto a un recuento inicial de neutrófilos absolutos menor de $1500 \mathrm{cel} / \mathrm{mm}^{3}$, con evidencia de descenso, en los siguientes 2 días consecutivos, a recuentos menores de $500 \mathrm{cel} / \mathrm{mm}^{3}$.

Se emplearon los antibiogramas reportados en la historia del laboratorio de microbiología de la institución. Las pruebas de identificación bioquímica y de susceptibilidad a los antimicrobianos fueron realizadas con el sistema automatizado Dade Behring Micros$\mathrm{can}^{\circledR}$ y el debido control de calidad microbiológico. La clasificación de microorganismo resistente se realizó teniendo en cuenta la Concentración Mínima Inhibitoria $(\mathrm{CMI})$ y la interpretación realizada por el laboratorio de acuerdo a los puntos de corte de las recomendaciones del Instituto de Estándares Clínicos y de Laboratorio (CLSI, por su sigla en inglés).

Se consideraron microorganismos clínicamente significativos, aquellos cuyo aislamiento correspondió a pacientes con sintomatología asociada. De tal modo, los microorganismos aislados en hemocultivos con significado clínico correspondieron a los que cumplieron con los criterios del Center for Disease Control and Prevention (CDC, 2018, Anexo 1) para infección del torrente sanguíneo ${ }^{5}$. Los microorganismos aislados en urocultivos y coprocultivos con significado clínico correspondieron a pacientes con sintomatología urinaria y gastrointestinal, respectivamente, en el momento de la obtención de la muestra.

Finalmente, se realizó una lectura interpretada de los antibiogramas de los cultivos positivos, para identificar el patrón fenotípico de resistencia antimicrobiana de las principales bacterias causantes de infecciones en la atención intrahospitalaria. Se consideró fenotipo de resistencia antimicrobiana a la combinación de un agente bacteriano causal y la resistencia a determinado antibiótico. Las definiciones de los patrones interpretados de resistencia antimicrobiana se encuentran en el anexo 2.
Basados en la definición de la OMS, los microorganismos se clasificaron en multidrogorresistentes (MDR), al presentar resistencia a 3 o más clases de agentes antimicrobianos disponibles en la mayor parte del mundo y que se consideran potencialmente eficaces contra el patógeno respectivo, y no-MDR para aquellos que no cumplían dichos criterios; para poder establecer su comportamiento en la población estudiada. Se consideraron fenotipos no-MDR, los CGP meticilino-sensibles y meticilino-resistentes, los BGN multisensibles, patrón de penicilinasa de bajo techo y alto techo. Se consideraron fenotipos MDR, los CGP Vancomicino-resistentes, BGN con patrón productor BLEE, con patrón PC y con patrón de MDR por mecanismos combinados (MDRmc) $)^{6,7}$.

El estudio fue considerado sin riesgo, aprobado por el comité de investigación de la Universidad Industrial de Santander (UIS) y de la CMISL; en el que se aplicó consentimiento y asentimiento informado verbal. No hay conflictos de interés.

Se realizó un análisis descriptivo de las medidas epidemiológicas de incidencia, mortalidad, letalidad, así como descripción de las variables por nivel de pacientes, episodios, cultivos, bacteriemia e interpretación de antibiograma. Para el análisis bivariado se usaron los test de chi2 de Pearson para variables categóricas, test exacto de Fisher para variables categóricas con bajo número y test de Mann-Whitney para variables cuantitativas continuas. En todos los análisis, se consideró un valor de $\mathrm{P}<0,05$ como estadísticamente significativo, con un intervalo de confianza de $95 \%$, en el programa Stata 14,0.

\section{Resultados}

De 1423 pacientes susceptibles, 130 cumplieron criterios de inclusión (1240 no presentaron NF; 48 no cumplieron criterios completos de NF, 2 cumplían criterios de exclusión según la metodología, 3 no firmaron consentimiento informado). En el periodo estudiado, se identificaron 315 episodios de NF clasificados de alto riesgo, hasta 9 episodios por paciente, para una mediana de 2,42 episodios por paciente y una incidencia acumulada de pacientes con primer episodio de NF de 9,1 ( IC $_{95 \%}$ 7,68-10,75). La mortalidad del periodo acumulado fue de 1,96 fallecidos por pacientes susceptibles, con una letalidad de 21,53 muertes por pacientes con NF durante todo el periodo.

Del total de episodios de NF, se obtuvieron 1454 cultivos, oscilando entre 1 y 33 cultivos por episodio, con una mediana de 4 (RIQ 3-5); fueron positivos $14,7 \%\left(\mathrm{n}=214 ; \mathrm{IC}_{95 \%} 13,0-16,6\right)$, para un total de 110 episodios con cultivos positivos y 220 microorganismos aislados (202 cultivos reportaron un solo agente; 
9 cultivos, dos agentes). En la tabla 1 se muestran las características de los pacientes estudiados.

El $62,2 \%$ de los episodios al ingreso presentaron foco infeccioso clínico, siendo los más frecuentes el respiratorio ( $\mathrm{n}=104 ; 33,1 \%$, ) y el gastrointestinal ( $\mathrm{n}=88 ; 28,1 \%)$. No hubo compromiso de sistema nervioso central en ninguno de los casos. Todos los niños recibieron antibiótico empírico inicial; los más usados como primera línea fueron TZP $(\mathrm{n}=153 ; 48,6 \%)$, FEP $(\mathrm{n}=118 ; 37,5 \%)$ y VAN $(\mathrm{n}=68 ; 21,6 \%)$.

Dentro de los tipos de cultivos realizados, los más frecuentes fueron hemocultivos $(\mathrm{n}=877 ;[60,3 \%], 109$ positivos), urocultivos ( $\mathrm{n}=355[24,4 \%], 17$ positivos $)$ y coprocultivos ( $\mathrm{n}=124 ;[8,5 \%], 53$ positivos).

De los aislamientos, 91,8\% correspondieron a bacterias, con predominio de BGN, seguidos de CGP, 7,3\% a hongos, $0,5 \%$ a parásitos y el restante a $\mathrm{Myco-}$ bacterium tuberculosis. Los microorganismos específicos aislados predominantes en orden descendente de frecuencia fueron: Klebsiella pneumoniae, Escherichia coli, SCN, Pseudomonas aeruginosa, Streptococcus viridans y Staphylococcus aureus. De los BGN aislados, casi el $90 \%$ fueron EB, la K. pneumoniae predominó en un poco más del $40 \%$ de los casos. Mientras que, en los CGP, los SCN fueron casi la mitad de los aislamientos. Respecto a los hongos, predominó la especie Candida tropicalis. No se obtuvieron cultivos para anaerobios y tampoco se contó con tecnología para la detección de virus. En la figura 1 se describen los microorganismos específicos aislados.

Al aplicarse los criterios del CDC, 38 pacientes presentaron infección del torrente sanguíneo, correspondientes a 55 episodios (17,5\% [55/315] del total de episodios y 50,5\% [55/109] de los episodios con cultivos positivos). De estos últimos, $9,1 \%(n=5 / 55)$ presentaron más de una infección del torrente sanguíneo durante el episodio, para un total de 60 bacteriemias (62 microorganismos). Entre tanto, en 14 ( $\mathrm{n}=14 / 315$, $4,4 \%)$ y $52(\mathrm{n}=52 / 315,16,5 \%)$ episodios se obtuvieron microorganismos con significado clínico en urocultivos y coprocultivos, respectivamente. La tabla 2 describe los diferentes microorganismos aislados en hemocultivos, coprocultivos y urocultivos.

Del total de aislamientos, $86,8 \%(\mathrm{n}=191)$ reportaron antibiograma. No en todos los casos se informó la resistencia para los antibióticos de acuerdo al microor-

\begin{tabular}{|c|c|c|}
\hline Variables & Valores absolutos & Medida (IC > 95\% o RIQ) \\
\hline Femenino/Masculino & $63 / 67$ & $48,46 \% / 51,54 \%$ \\
\hline Edad (años) & $3-16$ & 6,3 (RIQ: 3,0-10,0) \\
\hline Tumor hermatolinfoide & 108 & $83,08 \%(77,52-88,65)$ \\
\hline LLA & 77 & $59,23 \%(50,47-67,44)$ \\
\hline LMA & 16 & $12,31 \%(6,82-23,51)$ \\
\hline Linfomas no Hodgkin & 14 & $10,78 \%(3,64-39,51)$ \\
\hline Linfomas Hodgkin & 1 & $0,77 \%(0,11-5,4)$ \\
\hline Tumores sólidos & 18 & $13,85 \%(8,85-21,01)$ \\
\hline Otras enfermedades & 4 & $3,08 \%(0,84-12,39)$ \\
\hline Portador de Implantable & 58 & $45,08 \%(39,64-50,64)$ \\
\hline Profilaxis & 22 & $16,92 \%(10,27-17,93)$ \\
\hline Antibiótico últimos 3 meses & 163 & $51,75 \%(46,18-57,25)$ \\
\hline Comorbilidades & 98 & $31,11 \%(26,22-36,47)$ \\
\hline Días entre quimioterapia y fiebre & $0-40$ & 10 (RIQ: 4-13) \\
\hline Temperatura $\left({ }^{\circ} \mathrm{C}\right)$ & $38,0-40,5$ & 38,5 (RIQ: 38,2-38,9) \\
\hline Recuento de NA al inicio & $0-1419$ & 80 (RIQ: 14-280) \\
\hline Recuento de NA mínimo & $0-470$ & 20 (RIQ: 0-90) \\
\hline Días de neutropenia severa & $1-58$ & 7 (RIQ: 4-10) \\
\hline PCR al inicio del episodio & $6-396$ & 48 (RIQ: 24-192) \\
\hline Procalcitonina & $0,13-18,2$ & 1,49 (RIQ: 0,38-8,7) \\
\hline
\end{tabular}

Comorbilidades: Síndrome de Down y otras cromosomopatías, cardiopatías congénitas, neumopatías crónicas, desnutrición, hipotiroidismo, epilepsia, retraso psicomotor, insuficiencia renal, trastornos electrolíticos. Abreviatura: LLA: Leucemia linfoide aguda, LMA: Leucemia mieloide aguda, C: grados centígrados, AB: Antibiótico, NA: Neutrófilos absolutos, PCR: Proteína C Reactiva. 


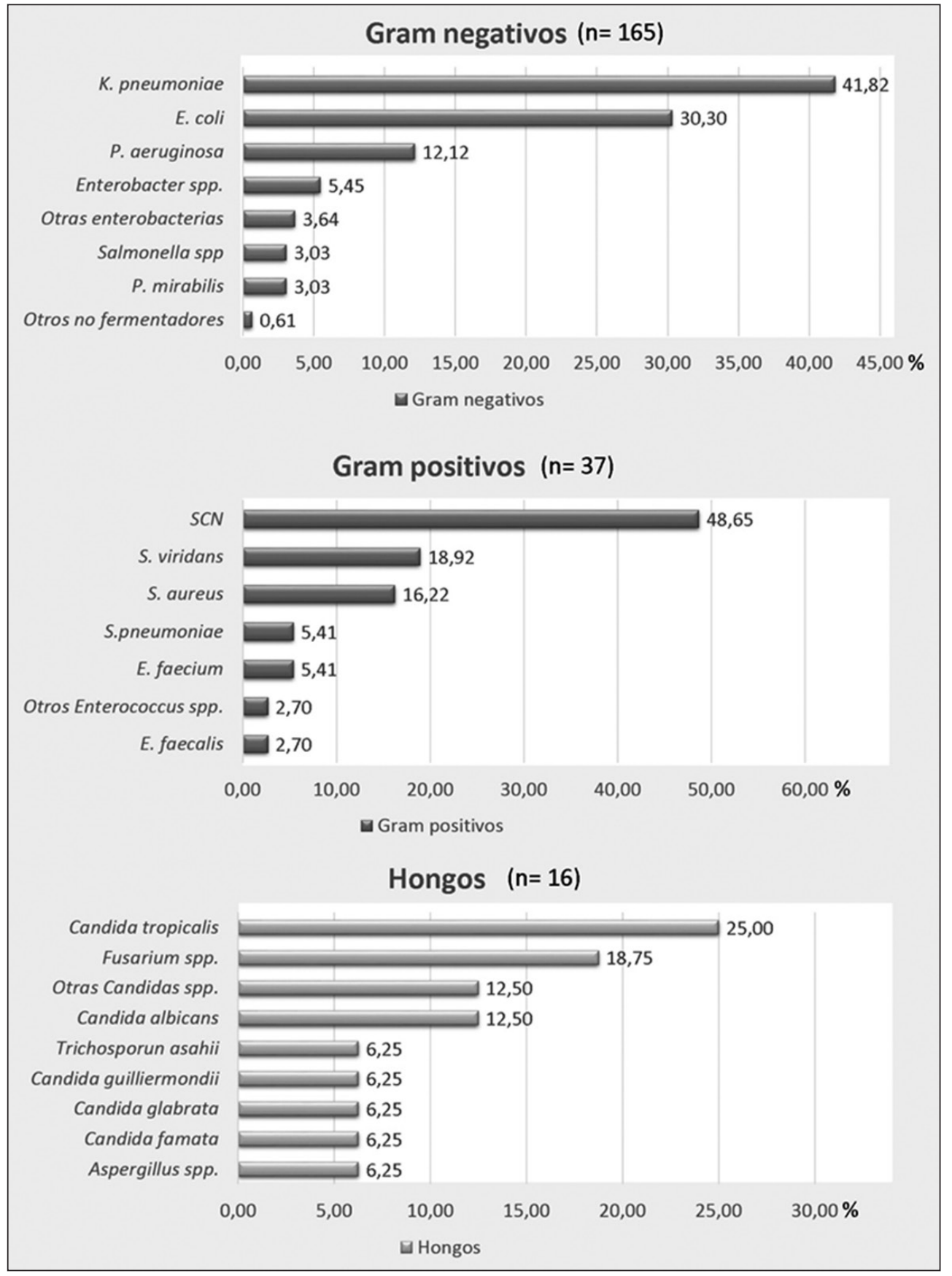

Figura 1. Microorganismos específicos aislados en el estudio. ganismo y no se reportó antibiograma en el género Streptococcus spp. La tabla 3 detalla la resistencia antibiótica por microorganismo específico de importancia clínica por su frecuencia y patogenicidad. De las EB, $40,46 \%$ mostraron resistencia a TZP, 33,34\% a FEP y $8,15 \%$ a MEM.

Respecto a los BGN, casi $60 \%$ de las EB fueron de fenotipos no-MDR. Dentro de los fenotipos MDR, se observó que una cuarta parte de las EB tienen patrón productor BLEE, con aparición de brotes del patrón sugestivo de PC. De los BGN no fermentadores $60 \%$ fueron multisensibles y 30\% presentaron fenotipos MDR al revelarse $10 \%$ con patrón sugestivo de PC y 20\% con patrón de MDRmc.

Con relación a los CGP, 10\% de los SCN fueron Meticilino-sensibles y $84 \%$ Meticilino-resistentes; mientras casi $70 \%$ de S. aureus fueron Meticilino-sensibles y un tercio Meticilino-resistentes (SAMR), estos últimos de fenotipo hospitalario. En el 2017, se aisló un SCN con CIM $\geq 2$ para Vancomicina, sensible a Linezolid en un hemocultivo central de un adolescente masculino con Leucemia mieloide aguda en recaída 
Tabla 2. Microorganismos aislados clínicamente significativos en los principales cultivos realizados en el estudio.

\begin{tabular}{|c|c|c|c|c|}
\hline Tipo & Microorganismo & $\begin{array}{l}\text { Frecuencia } \\
\text { (n) }\end{array}$ & $\begin{array}{l}\text { Porcentaje según tipo de } \\
\text { microorganismos } \\
(\%)\end{array}$ & $\begin{array}{c}\text { Porcentaje según total de } \\
\text { microorganismos aislados } \\
(\%)\end{array}$ \\
\hline \multicolumn{5}{|c|}{ Microorganismos aislados en Hemocultivos ( $n=62$ ) } \\
\hline \multirow{4}{*}{$\begin{array}{l}\text { Gram negativos } \\
(n=45,72,58 \%)\end{array}$} & Klebsiella pneumoniae & 19 & 42,18 & 30,65 \\
\hline & Escherichia coli & 15 & 33,33 & 24,19 \\
\hline & Pseudomonas aeruginosa & 8 & 17,76 & 12,90 \\
\hline & Otros BGN aislados ${ }^{\mathrm{a}}$ & 3 & 6,67 & 4,84 \\
\hline \multirow{4}{*}{$\begin{array}{l}\text { Gram positivos } \\
(\mathrm{n}=11,17,74 \%)\end{array}$} & Staphylococcus epidermidis & 4 & 36,36 & 6,45 \\
\hline & Staphylococcus aureus & 3 & 30,00 & 4,84 \\
\hline & Streptococcus mitis/oralis & 2 & 18,18 & 3,23 \\
\hline & Otros CGPb & 2 & 18,18 & 3,23 \\
\hline \multirow{3}{*}{$\begin{array}{l}\text { Hongos } \\
(n=6,9,68 \%)\end{array}$} & Candida tropicalis & 2 & 33,33 & 3,23 \\
\hline & Otras Candida spp. ${ }^{c}$ & 3 & 50,00 & 4,84 \\
\hline & Fusarium spp. & 1 & 16,67 & 1,61 \\
\hline \multicolumn{5}{|c|}{ Microorganismos aislados en Coprocultivos $(n=53)$} \\
\hline Gram negativos & Klebsiella pneumoniae & 15 & 31,25 & 28,30 \\
\hline \multirow[t]{7}{*}{$(n=48,90,57 \%)$} & Escherichia coli & 15 & 31,25 & 28,30 \\
\hline & Proteus mirabilis & 4 & 8,33 & 7,55 \\
\hline & Enterobacter cloacae & 3 & 6,25 & 5,66 \\
\hline & Enterobacter spp. & 2 & 4,17 & 3,77 \\
\hline & Salmonella spp. & 2 & 4,17 & 3,77 \\
\hline & Pseudomonas aeruginosa & 2 & 4,17 & 3,77 \\
\hline & Otros BGN aislados ${ }^{a}$ & 5 & 10,42 & 8,06 \\
\hline Hongos & Candida albicans & 2 & 50,00 & 3,77 \\
\hline$(n=4,7,55 \%)$ & Otras Candida spp.c & 2 & 50,00 & 3,77 \\
\hline $\begin{array}{l}\text { Parásitos } \\
(n=1,1,89 \%)\end{array}$ & Giardia lamblia & 1 & 100,00 & 1,89 \\
\hline \multicolumn{5}{|c|}{ Microorganismos aislados en Urocultivos $(n=14)$} \\
\hline Gram negativos & Klebsiella pneumoniae & 6 & 46,15 & 42,86 \\
\hline \multirow[t]{2}{*}{$(n=13,92.86 \%)$} & Escherichia coli & 6 & 46,15 & 42,86 \\
\hline & Raoultella (K.) ornithinolytica & 1 & 7,69 & 7,14 \\
\hline $\begin{array}{l}\text { Hongos } \\
(n=1,7,14 \%)\end{array}$ & Candida tropicalis & 1 & $100,00 \%$ & 7,14 \\
\hline
\end{tabular}

aOtros BGN en hemocultivos: Salmonella spp, Enterobacter cloacae, Enterobacter aerogenes. En coprocultivos: Citrobacter spp, Enterobacter aerogenes, Kluyvera ascorbata, Providencia alcalifaciens, Raoultella (K.) ornithinolytica. 'Otros CGP: Streptococcus pneumoniae, Enterococcus faecium. 'Otras Candida: Candida famata, Candida guilliermondii, Candida glabrata, Candida spp.

tardía y NF quien se mantuvo aislado y recibió tratamiento dirigido con cultivos posteriores negativos. Se documentaron pocos casos de Enterococcus spp., todos sensibles a Vancomicina. En la tabla 4 se expone la frecuencia de los patrones interpretados de resistencia antimicrobiana clínicamente significativos de los microorganismos aislados.

Dado el predominio de EB, se trazó la tendencia de los patrones de resistencia antimicrobiana durante el periodo estudiado, expuesta en la figura 2. Se observó un descenso de los patrones AmpC, No-MDR y BLEE, aunque con circulación permanente de estos dos últimos y brotes de microorganismos con patrón sugestivo de PC.

También se realizó una comparación global entre las EB multisensibles con las de patrón BLEE y PC. Hubo diferencia significativa entre estos grupos respecto al uso de antibiótico previo, neutropenia prolongada, neutropenia profunda y días de UCI, como se expone en la tabla 5. Esto último corresponde a un análisis global que invita a profundizar con estudios posteriores. 
Tabla 3. Resistencia antibiótica de los microorganismos específicos de relevancia clínica en el estudio

\begin{tabular}{|c|c|c|c|}
\hline \multicolumn{4}{|l|}{ Gram-negativos } \\
\hline Antibiótico & $\begin{array}{c}\text { K. pneumoniae }(\mathrm{n}=69) \\
\mathrm{n}(\%)\end{array}$ & $\begin{array}{c}\text { E. coli }(\mathrm{n}=20) \\
\mathrm{n}(\%)\end{array}$ & $\begin{array}{c}\text { P. aeruginosa }(n=20) \\
n(\%)\end{array}$ \\
\hline Ampicilina & $51 / 54(94,44)^{a}$ & $39 / 45(86,67)$ & - \\
\hline C1G & $27 / 40(67,50)$ & $15 / 41(48,39)$ & - \\
\hline TZP & $36 / 65(55,38)$ & $10 / 47(25,53)$ & $3 / 19(15,79)$ \\
\hline Cefoxitina & $8 / 63(12,70)$ & $8 / 46(17,39)$ & - \\
\hline Aztreonam & $23 / 53(43,40)$ & $7 / 43(16,28)$ & $5 / 17(33,33)$ \\
\hline C3G & $29 / 63(46,03)$ & $10 / 43(23,26)$ & - \\
\hline Cefepime & $29 / 65(44,62)$ & $10 / 47(21,28)$ & $6 / 19(31,58)$ \\
\hline Gentamicina & $15 / 65(23,08)$ & $10 / 47(21,28)$ & $9 / 19(47,37)$ \\
\hline Amikacina & $1 / 64(1,56)$ & $5 / 48(10,42)$ & $6 / 19(31,58)$ \\
\hline Ciprofloxacina & $24 / 65(36,92)$ & $8 / 45(17,78)$ & $5 / 19(26,32)$ \\
\hline Meropenem & $9 / 64(14,06)$ & $2 / 47(4,26)$ & $6 / 19(31,58)$ \\
\hline Ertapenem & $8 / 64(12,50)$ & $2 / 47(4,26)$ & - \\
\hline \multicolumn{4}{|l|}{ Gram-positivos } \\
\hline Antibiótico & $\begin{array}{c}\text { S. aureus }(n=6) \\
n(\%)\end{array}$ & $\begin{array}{c}\operatorname{SCN}(n=20) \\
n(\%)\end{array}$ & $\begin{array}{c}\text { Enterococcus }(n=4) \\
n(\%)\end{array}$ \\
\hline Penicilina & $4 / 5(80,00)^{a}$ & $2 / 12(16,66)^{a}$ & $3 / 4(75,00)$ \\
\hline Oxacilina & $2 / 5(40,00)$ & $16 / 18(88,89)$ & - \\
\hline Ampicilina & $4 / 5(80,00)^{a}$ & $10 / 10(100,00)^{\mathrm{a}}$ & $3 / 4(75,00)$ \\
\hline SAM & $2 / 5(40,00)$ & $12 / 14(85,71)$ & - \\
\hline Eritromicina & $2 / 5(40,00)$ & $2 / 16(12,50)$ & - \\
\hline Gentamicina & $1 / 5(20,00)$ & $9 / 15(60,00)$ & $2 / 2(100,00)$ \\
\hline Clindamicina & $2 / 5(40,00)$ & $13 / 18(72,22)$ & - \\
\hline Vancomicina & $0 / 5(0,00)$ & $1 / 18(5,56)$ & $0 / 4(0,00)$ \\
\hline TMP-SMX & $2 / 5(40,00)$ & $10 / 17(58,82)$ & - \\
\hline Linezolid & $0 / 5(0,00)$ & $0 / 16(0,00)$ & $2 / 4(50,000)$ \\
\hline
\end{tabular}

En cada celda el denominador corresponde al número de reportes de la resistencia del microorganismo al antibiótico específico. Corresponden a patrones de resistencia esperables (natural o adquirido): Klebsiella pneumoniae es naturalmente resistente a Ampicilina y los Staphylococcus spp. son resistentes adquiridos, mediante betalactamasa BLAZ, a las penicilinas y amonipenicilinas en un 80-90\% de los casos. Abreviatura: C1G: Cefalosporinas de primera generación, TZP: Piperacilina Tazobactam, C3G: Cefalosporinas de tercera generación. SAM: Ampicilina Sulbactam, TMP-SMX: Trimetoprim Sulfametoxazol.

\section{Discusión}

Se registró aislamiento microbiológico en el 35\% de los episodios, obtenidos mediante cultivos, dato consistente con los encontrados en la literatura $(1,2)$. Las bacterias continúan siendo la principal causa de infección documentada durante los episodios de NF, correspondiendo al $92 \%$ de los aislamientos en este estudio y hasta en el $60 \%$ en estudios previos, siendo más frecuentes los BGN con preponderancia de EB en casi el $90 \%^{1-3,8-10}$. La $K$. pneumoniae predominó en los BGN aislados, mientras que los $\mathrm{SCN}$ en los CGP aislados.

Los virus, aunque no fueron reportados, son la principal causa benigna de fiebre en pacientes con NF y su detección mediante técnicas moleculares es cada vez más común ${ }^{11,12}$. Si bien los hongos fueron menos frecuentes; la especie Candida tropicalis cobró importancia como infección asociada al cuidado de la salud en los pacientes con NF prolongada y recurrente, posiblemente al ser comensal de la barrera mucocutánea alterada en esta población ${ }^{13,14}$. La profilaxis con Fluco- 
Tabla 4. Frecuencia de los patrones interpretados de resistencia antimicrobiana de los microorganismos aislados en los episodios de NF del estudio ${ }^{a}$

\begin{tabular}{|c|c|c|c|c|}
\hline Patrón interpretado & $\begin{array}{l}\text { Frecuencia } \\
\text { (n) }\end{array}$ & $\begin{array}{l}\text { Porcentaje según } \\
\text { microorganismo }\end{array}$ & $\begin{array}{l}\text { Porcentaje del total } \\
\text { de aislamientos } \\
(n=220)\end{array}$ & $\begin{array}{l}\text { Porcentaje del total } \\
\text { de episodios } \\
(n=315)\end{array}$ \\
\hline No antibiograma & 29 & $100,00 \%$ & $13,18 \%$ & $9,20 \%$ \\
\hline \multicolumn{5}{|c|}{ Staphylococcus aureus ( $\mathrm{n}=6,2,64 \%$ ) } \\
\hline SA-MS & 4 & $66,67 \%$ & $1,82 \%$ & $1,27 \%$ \\
\hline SA-MR fenotipo hospitalario & 2 & $33,33 \%$ & $0,91 \%$ & $0,63 \%$ \\
\hline \multicolumn{5}{|c|}{ Staphylococcus coagulasa negativos ( $n=19,8,68 \%$ ) } \\
\hline SCN-MR & 16 & $84,21 \%$ & $7,27 \%$ & $5,08 \%$ \\
\hline SCN-MS & 2 & $10,53 \%$ & $0,91 \%$ & $0,63 \%$ \\
\hline SCN-VR & 1 & $5,26 \%$ & $0,45 \%$ & $0,32 \%$ \\
\hline \multicolumn{5}{|l|}{ Enterococcus spp $(\mathrm{n}=3,1,37 \%)$} \\
\hline E-AR & 2 & $66,67 \%$ & $0,91 \%$ & $0,63 \%$ \\
\hline E-AS & 1 & $33,33 \%$ & $0,45 \%$ & $0,32 \%$ \\
\hline \multicolumn{5}{|c|}{ Enterobacterias $(n=143,65,30 \%)$} \\
\hline EB-MS & 51 & $35,66 \%$ & $23,18 \%$ & $16,19 \%$ \\
\hline EB-BLEE & 36 & $25,17 \%$ & $16,36 \%$ & $11,43 \%$ \\
\hline EB-BLEE Kp & 21 & $14,66 \%$ & $9,55 \%$ & $6,67 \%$ \\
\hline EB-BLEE EC & 8 & $5,59 \%$ & $3,64 \%$ & $2,54 \%$ \\
\hline EB-PPasa de bajo techo & 21 & $14,66 \%$ & $9,55 \%$ & $6,67 \%$ \\
\hline EB-PPasa de alto techo & 12 & $8,39 \%$ & $5,45 \%$ & $3,81 \%$ \\
\hline EB-AmpC & 12 & $8,39 \%$ & $5,45 \%$ & $3,81 \%$ \\
\hline EB-PC ${ }^{b}$ & 11 & $7,69 \%$ & $5,00 \%$ & $3,49 \%$ \\
\hline \multicolumn{5}{|c|}{ Bacilos Gram-negativos no fermentadores ( $n=20,9,13 \%)$} \\
\hline BNF-MS & 12 & $60,00 \%$ & $5,45 \%$ & $3,81 \%$ \\
\hline BNF-MDRmc & 4 & $20,00 \%$ & $1,82 \%$ & $1.27 \%$ \\
\hline BNF-CS & 2 & $10,00 \%$ & $0,91 \%$ & $0,63 \%$ \\
\hline BNF-PC & 2 & $10,00 \%$ & $0,91 \%$ & $0,63 \%$ \\
\hline
\end{tabular}

a Las definiciones de los patrones interpretados de resistencia antimicrobiana se encuentran en Anexo 2. ${ }^{b}$ El $2,10 \%(n=3)$ fueron confirmados por los test de ácido borónico y Test carba NP. Abreviatura: SA-MS: Staphylococcus aureus Meticilino-sensible. SA-MR: Staphylococcus aureus Meticilino-resistente. E-AR: Enterococcus Ampicilina-resistente, E-AS: Enterococcus Ampicilina-sensible. EB-MS: Enterobacteria multisensible, EB-BLEE: Enterobacteria patrón productor de Betalactamasa de espectro extendido. Kp: Klebsiella pneumoniae. Ec: Escherichia coli. EB-PC: Enterobacteria patrón sugestivo de producción de carbapenemasas. BNF: Bacilos Gram-negativos no fermentadores. BNF-MS: BNF multisensible, BNF-MDRmc: BNF patrón sugestivo de Multidrogorresistencia mecanismos combinados, BNF-CS: BNF sensible a cefalosporina de 4ta generación, BNF-PC: BNF patrón sugestivo de producción de carbapenemasas.

Tabla 5. Variables asociadas a patrones de resistencia inusuales de EB aisladas en los episodios de NF en el estudio

\begin{tabular}{|c|c|c|c|c|}
\hline Variable & $\begin{array}{l}\text { Multisensibles } \\
\begin{array}{c}(n=51) \\
n(\%)\end{array}\end{array}$ & $\begin{array}{l}\text { Fenotipo BLEE } \\
\begin{array}{c}(n=36) \\
n(\%)\end{array}\end{array}$ & $\begin{array}{c}\text { Fenotipo EPC } \\
(n=11) \\
n(\%)\end{array}$ & Valor de $\mathrm{p}$ \\
\hline Uso de antibiótico previo & $26(50,98)$ & $30(83,33)$ & $2(18,18)$ & $<0,001$ \\
\hline Uso de dispositivos invasivos & $26(50,98)$ & $19(52,78)$ & $5(45,45)$ & 0,698 \\
\hline Neutropenia prolongada & $14(31,11)$ & $20(76,92)$ & $11(100,00)$ & $<0,001$ \\
\hline Neutropenia profunda & $44(86,27)$ & $36(100,00)$ & $11(100,00)$ & 0,021 \\
\hline Estancia en UCI & $18(35,29)$ & $13(36,11)$ & $4(36,36)$ & 0,077 \\
\hline Días de $\mathrm{UCl}^{\mathrm{a}}$ & $8,5(4-13)$ & $23(4-23)$ & $1(1-1)$ & 0,005 \\
\hline
\end{tabular}

Se presentan resultados por filas. ${ }^{a}$ Mediana (RIQ). 


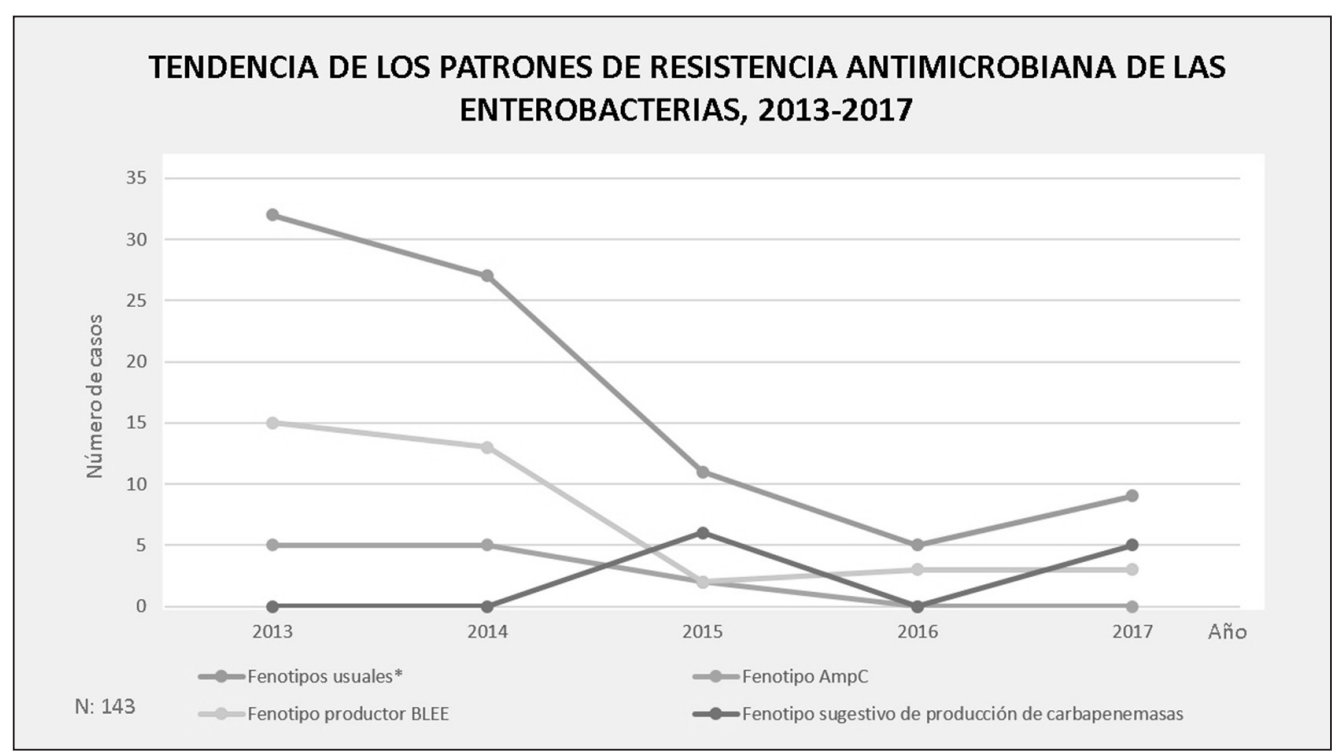

Figura 2. Tendencia de los patrones de resistencia antimicrobiana de las EB aisladas por episodios de NF en el estudio. nazol ha incrementado la presencia de otras especies resistentes a la misma, son ejemplos la Candida krusei y Candida glabrata ${ }^{15,16}$.

Respecto a los patrones de resistencia, las EB de patrón multisensible fueron las más frecuente con más de la mitad de los aislamientos, seguido de los productores BLEE con una cuarta parte; para los BGN no fermentadores predominó el patrón multisensible seguido de los MDRmc. Para los CGP prevaleció la sensibilidad a la Vancomicina (sólo se reportó un caso resistente); la mayoría de $S$. aureus fueron Meticilino-sensibles; mientras los SCN predominantemente Meticilino-resistentes. Las infecciones por bacterias MDR son menos incidentes en niños. Sin embargo, la emergencia de agentes MDR, como EB con patrón BLEE, PC, especies Pseudomonas MDR y Enterococcus Vancomicino-resistente (ERV), es un problema de salud pública actual ${ }^{10}$.

Estos patrones de resistencia varían según el contexto epidemiológico de cada institución. En efecto, a nivel local Rueda et al. caracterizó la NF en los niños del Hospital Universitario de Santander del 2007 al 2008, con 35 episodios de NF de alto riesgo y 6 hemocultivos positivos, cuyos microorganismos descritos fueron multisensibles (K. pneumoniae multisensible, S. aureus Oxacilino-sensible, Acinetobacter baumannii sensible y Candida parapsilosis) ${ }^{17}$. Aunque la muestra fue escasa; es el único estudio en nuestra ciudad.

En el panorama nacional colombiano, para el 2018 fue publicado un estudio descriptivo de los pacientes oncológicos con choque séptico hospitalizados en el Instituto Nacional de Cancerología de Bogotá, donde predominaron los BGN (56,9\%), seguidos de los CGP (31\%), donde el patógeno más aislado fue K. pneumo$n i a e^{18}$. En un estudio previo en dicha institución los
CGP fueron predominantes, no reportaron resistencia a Vancomicina, 20\% de los S. aureus y $40 \%$ de los SCN eran Oxacilino-resistentes, en contraste con nuestro estudio donde se duplicó la frecuencia de microorganismos resistentes a Meticilina. Mientras en los BGN, $23 \%$ de E. Coli y 14\% de K. pneumoniae fueron patrón BLEE; y $12 \%$ de BGN fueron resistentes a carbapenemicos (Meropenem o Imipenem) ${ }^{19}$.

En Medellín, para el 2013 se publicó un estudio realizado en el Hospital Pablo Tobón Uribe donde los microorganismos más frecuentes fueron los BGN encabezados por E. coli y K. pneumoniae, seguidos de S. aureus. Respecto a la susceptibilidad bacteriana, el patrón BLEE se detectó en 7,6\% de los episodios (una cepa de K. pneumoniae y dos de E. coli) y solo hubo un episodio con resistencia a carbapenémicos. Para los CGP, los $S$. aureus multisensibles predominaron en una relación 3:1 respecto a los Meticilino-resistentes, ninguno fue resistente a Vancomicina ${ }^{20}$. Resultados muy similares quedaron expuestos en años anteriores en el Centro Hematológico Infantil de la Universidad de Antioquia y en el Hospital San Vicente de Paúl ${ }^{21}$. En contraste, Pasto reportó mayor aislamiento de CGP respecto a $\mathrm{BGN}$, encabezados por $S$. aureus y $\mathrm{SCN}^{22}$.

De forma similar en Latinoamérica, si bien han sido predominantes los BGN, se ha visto una transición microbiana asociada al desarrollo del país. En Cuba, un estudio del 2012 mostró que los microorganismos predominantes fueron los BGN, aunque de manera aislada encabezaban la lista los $\mathrm{SCN}^{23}$. Entretanto, en México quedó en evidencia dicha tendencia, ya que para el 2014, los microorganismos causales comúnmente aislados fueron $E$. coli, $P$. aeruginosa, SCN y K. pneumoniae $^{24}$; mientras para el 2019 hubo un predominio 
de CGP (S. aureus, S. viridans $)^{25}$. Por su parte Brasil reportó predominio de microorganismos encapsulados (S. pneumoniae, H. influenzae), S. aureus y $\mathrm{BGN}^{2}$.

En Chile, país donde se ha documentado ampliamente el comportamiento microbiológico en instituciones de referencia, un estudio de los agentes causales de bacteriemia en niños con cáncer y NF de alto riesgo en cinco hospitales de Santiago entre 2012 y 2015 encontró un predominio equivalente de BGN (46,6\%) y CGP $(45,1 \%)$, siendo E. coli el más frecuente, de la mano con SCN con una resistencia del $86,4 \%$ a Oxacilina (26). Cercano a lo reportado en los mismos centros entre 2004 y 2009, donde la frecuencia de aislamiento de los CGP fue predominante (56\%), siendo los agentes más frecuentes SCN, E. coli, S. viridans y $S$. aureus; con resistencia a Oxacilina en un $77 \%$ de SCN y $14 \%$ de S. aureus $^{27}$. Resultados similares fueron descritos por Cortez en 2008 y Ducasse en el 201428,29. Maldonado propone que esta similitud en las resistencias a pesar del paso de los años, corresponde al conocimiento riguroso de la epidemiología local que lleva al uso racional de antibióticos; en contraste con el incremento de las resistencias observado en otros países ${ }^{26}$.

En general, diversos estudios a nivel mundial (incluyendo países como Estados unidos, Alemania, Países Bajos, Italia, Australia, India, Israel y Egipto), concuerdan que existe una baja frecuencia de SAMR, con circulación importante de EB con patrón BLEE y emergencia de ERV, EPC y especies Pseudomonas spp. $\mathrm{MDR}^{13,30-35}$

El riesgo en esta población de presentar altos niveles de resistencia a los antibióticos responde a complejos factores sinérgicos intrínsecos del huésped, patógeno y ambiente (múltiples hospitalizaciones, mayor intervención al paciente, uso recurrente de antibioticoterapia empírica de forma inapropiada, comorbilidades asociadas, entre otros $)^{36,37}$.

En este estudio, el comportamiento de los microorganismos patógenos fue similar al reportado en otros países de bajos y medianos ingresos, donde las EB fueron predominantes, con aumento de microorganismos MDR. Este comportamiento pudiera obedecer a factores como: limitación en las políticas de control y prevención de infecciones, nuevos tratamientos de quimioterapia intensiva que incrementan el riesgo de translocación bacteriana de microorganismos entéricos por mucositis, presión selectiva favorecida por algunas profilaxis antibióticas, así como el no uso de profilaxis con fluoroquinolonas, entre otros ${ }^{14,38,39}$.

Por su parte, los países de altos ingresos tienen predominio de CGP, debido a la medicina intervencionista que, si bien ha favorecido el manejo oportuno y adecuado de la NF, consigo ha incrementado al S. aureus como principal causa de infección grave con progresión a choque séptico, al S. epidermidis como principal patógeno de las bacteriemias asociadas a catéteres centrales; y a la mucositis oral por quimioterapia intensiva que predispone a bacteriemias por S. viridans $^{3,40}$. Por lo anterior, la evaluación de los patrones de resistencia nos permite estudiar nuevas estrategias para el control de infecciones y el uso racional de los antibióticos según el contexto epidemiológico en el que nos encontramos.

\section{Fortalezas y Limitaciones}

Este trabajo es representativo de la población local al haberse realizado en una institución de referencia de la región y en un periodo de estudio amplio. La vasta población reflejada en este estudio permite que los resultados sean relevantes tanto a nivel nacional como en Latinoamérica. No hubo sesgo de selección dada la rigurosidad de la aplicación de los criterios de inclusión y exclusión. Sin embargo, debido a que fue un estudio descriptivo retrospectivo, podría existir sesgo de información.

\section{Conclusiones}

El comportamiento de los microorganismos y su patrón de susceptibilidad antimicrobiana varía en cada región y entre cada institución. En nuestro estudio se refleja que en pacientes pediátricos con patología hemato-oncológica y NF, en una institución de referencia en Colombia, existe un predominio de microorganismos Gram-negativos como agentes causales de infección, siendo más comunes las $\mathrm{EB}$, con una circulación importante de patrón BLEE y brotes de EPC, con niveles de resistencia similares a los observados en otros países de bajos y medianos ingresos.

\section{Responsabilidades Éticas}

Protección de personas y animales: Los autores declaran que los procedimientos seguidos se conformaron a las normas éticas del comité de experimentación humana responsable y de acuerdo con la Asociación Médica Mundial y la Declaración de Helsinki.

Confidencialidad de los datos: Los autores declaran que han seguido los protocolos de su centro de trabajo sobre la publicación de datos de pacientes.

Derecho a la privacidad y consentimiento informado: Los autores han obtenido el consentimiento informado de los pacientes y/o sujetos referidos en el artículo. Este documento obra en poder del autor de correspondencia. 


\section{Conflicto de intereses}

Los autores declaran no tener conflicto de intereses.

\section{Agradecimientos}

Nuestro sincero agradecimiento a la Clínica Materno
Infantil San Luis por autorizarnos y facilitarnos la realización del trabajo. Así mismo los autores reconocemos y agradecemos la colaboración en la recolección de datos a Valentina Gualdrón-Rincón, Maria Paula Silva-Sánchez, Slendy Bautista-Sanguino, Alba Anteliz-Díaz, Lorena Villamizar-Peña, Ahydaly GrandasHernández, así como la ayuda técnica de Laura Bonilla-Tinoco.

\section{Anexo 1. Criterios del Center for Disease Control and Prevention (2018) para infection del torrente sanguíneo}

- Criterio 1: Infección en paciente de cualquier edad en el que se documenta un organismo patógeno, en al menos uno de los especímenes de sangre obtenidos por hemocultivos o métodos microbiológicos no basados en cultivos realizados del torrente sanguíneo. No es necesario que existan signos o síntomas para cumplir con los criterios de infección del torrente sanguíneo confirmada por laboratorio.

- Criterio 2: Infección en paciente de cualquier edad que tiene al menos uno de los siguientes signos o síntomas: Fiebre (> $38^{\circ} \mathrm{C}$ ), escalofríos o hipotensión y en el que se identifica un microorganismo comensal en al menos dos especímenes de sangre recolectados en dos ocasiones diferentes que incluyen: difteroides (Corynebacterium spp. C. diphtheria), Bacillus spp. (B. anthracis), Propionibacterium spp., SCN (incluido S. epidermidis), S. viridans, Aerococcus spp. Micrococcus spp. y Rhodococcus spp.

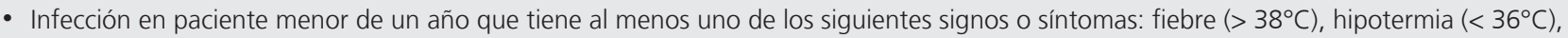
apnea o bradicardia, en el que se identifica un microorganismo comensal en dos o más muestras de sangre.

Center For Disease Control And Prevention. Bloodstream Infection Event (Central Line-Associated Bloodstream Infection and Non-central Line Associated Bloodstream Infection). [En línea]. Guidelines and recommendations. USA. CDC.gov. 2018. (Recuperado en 28 de Febrero 2019 ) Disponible en: https://www.cdc.gov/nhsn/pdfs/pscmanual/4psc_clabscurrent.pdf.

\begin{tabular}{|c|c|c|}
\hline Microorganismo & Patrón interpretado & Lectura interpretada \\
\hline Staphylococcus aureus & $\begin{array}{l}\text { SA-MS } \\
\text { SA-MR fenotipo comunitario } \\
\text { SA-MR fenotipo hospitalario } \\
\text { VI-SA } \\
\text { VR-SA }\end{array}$ & $\begin{array}{l}\text { S. aureus sensible a Meticilina/Oxacilina. } \\
\text { S. aureus resistente a Meticilina/Oxacilina, sensible a Clindamicina y TMP-S- } \\
\text { MX, Gentamicina. } \\
\text { S. aureus resistente a Meticilina/Oxacilina, Clindamicina, TMP-SMX, Gentami- } \\
\text { cina., sensible solo a Vancomicina. } \\
\text { S. aureus con resistencia intermedia a vancomicina. Requiere pruebas confir- } \\
\text { matorias. } \\
\text { S. aureus resistente a vancomicina. Requiere pruebas confirmatorias. }\end{array}$ \\
\hline $\begin{array}{l}\text { Staphylococcus coagulasa } \\
\text { negativo }\end{array}$ & $\begin{array}{l}\text { SCN-MS } \\
\text { SCN-MR } \\
\text { SCN-VR }\end{array}$ & $\begin{array}{l}\text { S. Coagulasa negativo sensible a Meticilina. } \\
\text { S. Coagulasa negativo resistente a Meticilina, sensible a Vancomicina. } \\
\text { S. coagulasa negativo resistente a Vancomicina. }\end{array}$ \\
\hline Enterococos & $\begin{array}{l}\text { E-AS } \\
\text { E-AR } \\
\text { E-RV }\end{array}$ & $\begin{array}{l}\text { Enterococos sensibles a Ampicilina. } \\
\text { Enterococos resistentes a Ampicilina y sensibles a Vancomicina. } \\
\text { Enterococos resistentes a Vancomicina. }\end{array}$ \\
\hline Estreptococos & $\begin{array}{l}\text { S-PS } \\
\text { S-PI } \\
\text { S-PR }\end{array}$ & $\begin{array}{l}\text { Estreptococos sensibles a Penicilina. } \\
\text { Estreptococos con resistencia intermedia a Penicilina, sensibles a Ceftriaxona } \\
\text { y Vancomicina. } \\
\text { Estreptococos resistentes a Penicilina, sensibles a Ceftriaxona y Vancomicina. } \\
\text { Estreptococos resistentes a Ceftriaxona y sensibles a Vancomicina. }\end{array}$ \\
\hline
\end{tabular}




\begin{tabular}{|c|c|c|}
\hline Enterobacterias & $\begin{array}{l}\text { EB-AmpC } \\
\text { EB-BLEE } \\
\text { EB-PC } \\
\text { EB-MDRmc }\end{array}$ & $\begin{array}{l}\text { Enterobacterias multisensibles. } \\
\text { (La K. pneumoniae puede ser naturalmente resistente a ampicilina). } \\
\text { EB con patrón sugestivo productor de penicilinasas: } \\
\text { - Penicilinasa de bajo techo: Resistente a Ampicilina, Piperacilina, Ampicilina- } \\
\text { - Sulbactam, cefalosporinas de primera y segunda generación. } \\
\text { - Penicilinasas de alto techo: Resistente a Ampicilina, Piperacilina, Ampicilina- } \\
\text {-Sulbactam, Piperacilina-Tazobactam, cefalosporinas de primera y segunda } \\
\text { generación. } \\
\text { EB con patrón sugestivo productor de AMPc (Resistente a cefalosporinas de } \\
\text { primera, segunda, tercera generación y Cefoxitin resistentes). } \\
\text { EB con patrón sugestivo productor de betalactamasas de espectro extendido } \\
\text { (Resistente a cefalosporinas de primera, segunda, tercera y cuarta generación, } \\
\text { Aztreonam resistente, pueden ser sensibles o resistentes a Cefoxitin). } \\
\text { EB patrón sugestivo productor de carbapenemasas (Resistente a carbapené- } \\
\text { micos: } 3 \text { o más carbapenémicos incluido Ertapenem, pueden tener comporta- } \\
\text { miento BLEE). Requiere pruebas de confirmación. } \\
\text { EB patrón sugestivo de patrón de multirresistencia de mecanismos combina- } \\
\text { dos (Bomba de eflujo, porinas, etc. Requiere lectura interpretada del antibio- } \\
\text { grama por infectóloga investigadora). }\end{array}$ \\
\hline $\begin{array}{l}\text { Bacilos Gram-Negativos no } \\
\text { fermentadores }\end{array}$ & $\begin{array}{l}\text { BNF-MS } \\
\text { BNF-CS } \\
\text { BNF-PC } \\
\text { BNF-MDRmC }\end{array}$ & $\begin{array}{l}\text { Bacilos Gram-Negativos no fermentadores multi-sensibles. } \\
\text { (Podría existir resistencia intrínseca a Cefalosporinas de primera, segunda y } \\
\text { tercera generación). } \\
\text { Microorganismos no fermentadores sensibles a cefalosporinas de cuarta } \\
\text { generación (sensibles a Cefepime) } \\
\text { Bacilos Gram-Negativos no fermentadores con patrón sugestivo de car- } \\
\text { bapenemasas: resistencia a } 3 \text { o más Carbapenémicos. Requiere pruebas de } \\
\text { confirmación. } \\
\text { Bacilos Gram-Negativos no fermentadores con patrón sugestivo de patrón de } \\
\text { multirresistencia por mecanismos combinados (Bomba de eflujo, porinas, etc. } \\
\text { Requiere lectura interpretada del antibiograma por infectóloga investigadora). }\end{array}$ \\
\hline
\end{tabular}

\section{Referencias}

1. Sociedad Argentina de Pediatría. Consensus on management of children with can-cer, neutropenia and fever. Update 2008-2009. Arch Argent Pediatr. 2010;108(2):e47-e70.

2. Mendes AV, Sapolnik R, Mendonça $\mathrm{N}$. New guidelines for the clinical management of febrile neutropenia and sepsis in pediatric oncology patients. J Pediatr (Rio J). 2007;83(Suppl2):S54-63.

3. Fisher B, Sung L. Chapter 68: The febrile neutropenic patient. In: Cherry J, Demmler-Harrison G, Kaplan S, Steinbach W, Hotez P, ed. by. Feigin and Cherry's Textbook of Pediatric Infectious Diseases. Houston, Texas, USA: Elsevier; 2018; 8:657-64.

4. Lehrnbecher T. Guideline for the Management of Fever and Neutropenia in Chil-dren With Cancer and Hematopoietic Stem-Cell Transplantation Recipients: 2017 Update. J Clin Oncol. 35:2082-94.
5. Center For Disease Control And Prevention. Bloodstream Infection Event (Central Line-Associated Bloodstream Infection and Non-central Line Associated Blood-stream Infection). [En línea]. Guidelines and recommendations. USA. CDC.gov. 2018. (Recuperado en 28 de Febrero 2019) Disponible en: https:// www.cdc.gov/nhsn/pdfs/pscmanual/4psc_ clabscurrent.pdf.

6. Jiménez Pearson MA, Galas M, Corso $\mathrm{A}$, et al. Consenso latinoamericano para definir, categorizar y noti-ficar patógenos multirresistentes, con resistencia extendida o panresistentes. Rev Panam Salud Publica. 2019;43:e65.

7. OMS-OPS. Prevención y control de infecciones asociadas a la atención en salud. Recomendaciones básicas. Capítulo V: Precauciones para prevenir infecciones por agentes multirresistentes y resistentes de importancia para la salud pública. 2017

8. Freifeld AG, Bow EJ, Sepkowitz KA, et al. Clinical practice guideline for the use of antimicrobial agents in neutropenic patients with cancer: 2010 update by the infectious diseases society of America. Clin Infect Dis. 2011;52(4):e56-93.

9. Santolaya ME, Farfán MJ, De La Maza V, et al. Diagnosis of bacteremia in febrile neutropenic episodes in children with cancer: microbiologic and molecular approach. Pediatr Infect Dis J. 2011;30(11):957-61.

10. Baro M. Predicción del riesgo de infección grave en niños con neutropenia febril se-cundaria a quimioterapia. [PhD thesis]. Madrid: Programa de Doctorado en Investi-gación en Ciencias MédicoQuirúrgicas Universidad Complutense de Madrid; 2017.

11. Rivera D, Valverde K, Ávila ML. Neutropenia febril en niños con cáncer: manejo en el servicio de emergencias. Revista chilena de infectología 2018; 35(1): 62-71.

12. Hakim H, Flynn PM, Knapp KM, Srivastava DK, Gaur AH. Etiology and clinical course of febrile neutropenia in children with cancer. Journal of pediatric 
hematolo-gy/oncology 2009; 31(9):623-9.

13. Paganini H, Santolaya ME, Álvarez M, et al. Diag-nóstico y tratamiento de la neutropenia febril en niños con cáncer. Consenso de la Sociedad Latinoamericana de Infectología Pediátrica. Rev Chil Infect 2011; 28 Supl 1:10-38.

14. Ramos J, Francisco L, Daoud Z. Infección fúngica invasora en niños: diferencias y homologías con el adulto. Rev Esp Quimioter. 2016;29 suppl 1: 59-65.

15. Lucero $Y$, Brucher R, Alvárez AM, et al. Infección micótica profunda en niños con cáncer, neutropenia y fiebre, en Chila. Rev Méd Chile. 2002;130(10):1139-46.

16. Santolaya ME, de Queiroz F, Alvarado $\mathrm{T}$, et al. Recommendations for the management of candidemia in children in Latin America. Rev Iberoam Micol. 2013;30(3):171-8.

17. Rueda E, Trujillo ML, Díaz LA. La neutropenia severa febril en niños con cáncer. Estudio descriptivo en el Hospital Universitario de Santander. Salud UIS. 2010;42(2):103-11.

18. Jiménez A, Godoy J, Vásquez P, Maya LC, Suárez A. Pacientes pediátricos con choque séptico que ingresan a la Unidad de Cuidado Intensivo Pediátrico del Insti-tuto Nacional de Cancerología. Revista Colombiana de Cancerología 2018;22(2):64-8.

19. Cortes JA, Cuervo SI, Arroyo P, Quevedo R. Hallazgos microbiológicos en pacientes con neutropenia febril. Rev Colomb Cancerol. 2003;7(4):5-11.

20. Madrid C, Díaz L, Combariza J, et al. Epidemiología de la neutropenia febril en pacientes adultos con neoplasia hematológica, en un pe-ríodo de 26 meses en el Hospital Pablo Tobón Uribe, Colombia. Rev Chil Infectol. 2013;30 (2):195-201.

21. Ramírez L, Blanco N, Hernández M, et al. Episodios de neutropenia febril en niños con neoplasias malignas. Infectio 2003;7:137-46.

22. Suárez DV, Àlvarez M, Gómez JF, Carrasco MM,Burbano DC. Caracterización clí-nica y de laboratorio de pacientes con neutropenia febril en un hospital pediátrico en Pasto-Colombia. Pediatría 2016;49(2):48-53.

23. Martell-Martorell L, Arencibia-Núñez A. Infecciones intrahospitalarias en pacientes pediátricos con enfermedades hematológicas, 2006-2009. Rev Cuba de Hematol, Inmunol y Hemoter. 2012; 28(3):253-62.

24. Avilés-Robles M, Ojha RP, González $\mathrm{M}$, et al. Bloodstream infections and inpatient length of stay among pedi-atric cancer patients with febrile neutropenia in Mexico City. Am J Infect Control. 2014;42(11):1235-7.

25. Pacheco-Rosasa D, Peregrino-Bejaranoa L, López-Aguilar J, Juan-Shumc L, Miranda-Novales M. Piperacilina/tazobactam más amikacina vs. piperacilina/ tazobactam: tratamiento en niños con neutropenia febril. Rev Med Inst Mex Seguro Soc. 2019;57(2):65-73.

26. Maldonado ME, Acuña M, Álvarez $\mathrm{AM}$, et al. Mi-croorganismos aislados de hemocultivos en niños con cáncer y neutropenia febril de alto riesgo en cinco hospitales de Santiago, Chile, período 2012-2015. Revista chile-na de infectología. 2018;35(2):140-6.

27. Solís Y, Álvarez AM, Fuentes D, et al. Agentes causantes de bacteriemia en niños con cáncer y neutropenia febril de alto riesgo en seis hospitales de Santiago, Chile, período 2004-2009. Rev Chil Infect. 2012;29(2):156-62.

28. Cortez D, Rodríguez N, Benadof D, Zamorano A, Tordecilla J. Bacteriemia en pacientes oncológicos. Experiencia en un hospital pediátrico. Rev Chil Infectol. 2012; 29(2):164-8.

29. Ducasse K, Fernández JP, Salgado C, et al. Caracterización de los episodios de neutropenia febril en niños con leucemia mieloide aguda y leucemia linfoblástica aguda. Rev. chil. infectol. 2014;31(3):3338.

30. El-Mahallawy HA, Hassan SS, El-Wakil M, Moneer MM, Shalaby L. Increasing Antimicrobial Resistance Monitored in Surveillance Analysis of Blood Stream Infections in Febrile Neutropenic Pediatric Oncology Patients. Asian Pac J Cancer Prev. 2015;16(14):5691-5.

31. Greenberg D, Moser A, Yagupsky P, et al. Microbiological spectrum and susceptibility patterns of pathogens causing bacteraemia in paediatric febrile neutropenic oncology patients: comparison between two con-secutive time periods with use of different antibiotic treatment protocols. Int J Antimicro Agents. 2005;25(6):469-73.

32. Miedema KG, Winter RH, Ammann RA, et al. Bacteria causing bacteremia in pediatric cancer patients presenting with febrile neu-tropenia--species distribution and susceptibility patterns. Support Care Cancer. 2013;21(9):2417-26.

33. Castagnola E, Caviglia I, Pescetto L, Bagnasco F, Haupt R, Bandettini R. Antibiotic susceptibility of Gramnegatives isolated from bacteremia in children with can-cer. Implications for empirical therapy of febrile neutropenia. Future Microbiol. 2015; 10(3): 357-65.

34. Haeusler GM, Mechinaud F, Daley AJ, et al. Antibiotic-resistant Gram-negative bacteremia in pediatric oncology patients-risk factors and outcomes. Pediatr Infect Dis J. 2013;32(7):723-6.

35. Siddaiahgari S, Manikyam A, Kumar KA, Rauthan A, Ayyar R. Spectrum of sys-temic bacterial infections during febrile neutropenia in pediatric oncology patients in tertiary care pediatric center. Indian J Cancer 2014;51(4):403-5.

36. Calitri C, Ruberto E, Castagnola E. Antibiotic prophylaxis in neutropenic children with acute leukemia: Do the presently available data really support this practice? Eur J Haematol. 2018 Dec;101(6):721-727.

37. Ford CD, Lopansri BK, Gazdik MA, et al. Room con-tamination, patient colonization pressure, and the risk of vancomycin-resistant En-terococcus colonization on a unit dedicated to the treatment of hematologic malignancies and hematopoietic stem cell transplantation. Am J Infect Control. 2016;44(10):1110-5.

38. Klastersky J, De Naurois J, Rolston K, et al. Management of febrile neutropenia: ESMO Clinical Practice Guidelines. Annals of Oncology 2016;27 Supl 5:v1118.

39. Manterola A, Romero P, Martínez E, et al. Neutropenia y fiebre en paciente con cáncer. An Sist Sanit Navar. 2004; 27 supl 3:33-43.

40. Kebudi R, Kizilocak H. Febrile Neutropenia in Children with Cancer: Approach to Diagnosis and Treatment. Current Pediatric Reviews 2018;14(3):204-9. 